Research Article

\title{
Microneedling in Combination with Topical Pimecrolimus $1 \%$ versus Topical Pimecrolimus 1\% for the Treatment of Refractory Stable Vitiligo: A Randomized Clinical Trial
}

\author{
Fariba Iraji $\left(\mathbb{D}\right.$, Ali Asilian $\left(\mathbb{D}\right.$, Zahra Talebzadeh $\left(\mathbb{D}\right.$, Mina Saber $\mathbb{D}$, Fatemeh Mokhtari $\mathbb{D}^{D}$, \\ Amirhossein Siadat $\mathbb{\complement}$, and Seyed Mohsen Hosseini \\ Department of Dermatology, School of Medicine Skin Disease and Leishmaniasis Research Center, \\ Isfahan University of Medical Sciences, Isfahan, Iran \\ Correspondence should be addressed to Ali Asilian; asilian@med.mui.ac.ir
}

Received 22 September 2021; Revised 2 November 2021; Accepted 12 November 2021; Published 30 November 2021

Academic Editor: Giuseppe Stinco

Copyright (c) 2021 Fariba Iraji et al. This is an open access article distributed under the Creative Commons Attribution License, which permits unrestricted use, distribution, and reproduction in any medium, provided the original work is properly cited.

\begin{abstract}
Objective. Vitiligo is a common, autoimmune disease that results in the destruction of the melanocytes and manifests as depigmented macules on various areas of the skin. Numerous treatment options have been proposed for vitiligo. The purpose of this study was to compare the efficacy of microneedling plus topical pimecrolimus $1 \%$ versus the sole use of topical pimecrolimus $1 \%$ for the treatment of vitiligo. Methods. This clinical trial was conducted on 30 skin lesions on $15 \mathrm{Al}$-Zahra hospital patients. Each patient had two similar lesions in the limb area, and each lesion was considered a separate treatment group. The left or right side of the patient's lesion was randomly assigned to receive microneedling plus topical pimecrolimus for three months, while the other side received only topical pimecrolimus $1 \%$. As part of the follow-up, digital photography was taken at the baseline and biweekly for three months after treatment and six months' follow-up. The following methods were used to evaluate the results: DLQI questionnaires, patient satisfaction questionnaires, and two independent dermatologists comparing the improvement rate for each group. Results. Topical pimecrolimus $1 \%$ treatment led to unsatisfactory results, whereas the combination of microneedling and topical pimecrolimus $1 \%$ treatment produced a more favorable overall outcome $(P<0.001)$. Conclusion. This study established that combination therapy results in more significant patient improvement. Additionally, one patient experienced mild skin irritation as a side effect of topical pimecrolimus.
\end{abstract}

\section{Introduction}

Vitiligo is a common skin disease that affects between $1 \%$ and $2 \%$ of the world population [1] that occurs equally in men and women and is most prevalent between the ages of 10 and 30 [2]. Although various explanations for the mechanism and etiology of vitiligo have been proposed, including genetics, autoimmune mechanisms, psychological factors, biochemical factors, oxidative stress, and viral infections, the exact cause of vitiligo remains unclear $[3,4]$.

Multiple factors contribute to the mechanism of vitiligo. Stress, physical and mental trauma, and prolonged exposure to UV light are all possible etiologies of vitiligo [5]. HX positivity is reported in $20 \%-30 \%$ of families [6]. Vitiligo is an autoimmune disease frequently associated with other autoimmune conditions, including hypothyroidism, hyperthyroidism, rheumatoid arthritis, and diabetes mellitus [2]. This disorder is frequently distressing in patients and imposes a cosmetic burden on them, impairing their quality of life, self-esteem, marriage, and employment status [7]. Vitiligo is disfiguring in all races, particularly in darkskinned people [8]. Vitiligo's basic pathogenesis is currently unknown [9].

While there is no cure for vitiligo, several recommendations include topical steroids, topical calcipotriol, surgery, topical and systemic PUVA, and multivitamins [10, 11]. Calcineurin inhibitors are also a treatment option that appears to have fewer side effects than steroids and are better 
suited to long-term use, particularly in sensitive areas [12]. Calcineurin inhibitors act as topical immunomodulators by blocking T-cell activation [13]. Microneedling was also used in some studies and was found to be effective in the repigmentation of lesions [14], by transdermal drug delivery as it increases the absorption of topical drugs [15]. Microneedling and topical pimecrolimus have distinct mechanisms of action, and previous research has indicated that combining microneedling with topical treatment may increase the treatment's effectiveness [16]. There has been no comparison of the effects of topical pimecrolimus and microneedling. Thus, this study was conducted to determine the effect of topical pimecrolimus and microneedling on refractory stable vitiligo when used alone or in combination.

\section{Materials and Methods}

2.1. Study Design. This study was performed as a randomized clinical trial on 32 lesions from 16 patients who were randomly selected from vitiligo patients who referred to dermatology clinics affiliated with Isfahan University of Medical Sciences between Dec. 2019 and Dec. 2020. After informing all patients of the trial's procedures, written informed consent was obtained, and the trial was initiated. Two similar lesions on two similar limb locations were chosen for each patient. The lesions were then randomly assigned the letters $\mathrm{A}$ and B. Lesion A was treated with topical pimecrolimus $1 \%$ plus microneedling concurrently, whereas lesion B was treated with topical pimecrolimus $1 \%$ solely. Each lesion was examined as a distinct entity. Patients were treated for three months; Microneedling was performed every two weeks, and topical cream was also used twice a day. Patients were then followed up for six months. This study was registered on the Iranian clinical trials registry website under the identification number IRCT20190521043664N. Additionally, this study was approved by Isfahan University of Medical Sciences' bioethics committee and assigned the approval code IR.MUI.MED.REC.1398.413.

The inclusion criteria were patients between the ages of 18 and 60, had vitiligo lesions on their limbs, did not respond to light therapy or topical or systemic treatments, and did not have active vitiligo or new lesions within the past year. The exclusion criteria were pregnancy or breastfeeding, vitiligenous lesions in sites other than extremities, poor compliance to the trial and follow-up, disease deterioration during the study, current or previous vitiligo treatment one year prior to enrollment, keloid tendency, systemic disease, and severe allergic reactions or side effects to the drugs used throughout the trial.

Initially, the inclusion criteria were used to evaluate and confirm the qualifications of 20 patients. Four patients were excluded from the study during the first stage because three were unwilling to participate and another was ineligible. Thus, 32 lesions were chosen from 16 patients with stable refractory vitiligo. Three months of treatment and six months of follow-up were required. One patient was excluded from the study after the first session due to personal reasons; hence, the final analysis included 30 lesions from 15 patients (Figure 1).
2.2. Procedure Steps. Lesions A was simultaneously treated with topical pimecrolimus $1 \%$ and microneedling, and topical treatment with pimecrolimus cream $1 \%$ was initiated twice daily and continued for three months for lesions classified as B. Microneedling was used to treat these lesions every two weeks, and topical pimecrolimus $1 \%$ was applied immediately to the lesion under an occlusive dressing. The amount of topical pimecrolimus ointment was such that it covered the surface of the lesions in a thin layer. After 48 hours, patients were instructed to remove the dressing and apply topical pimecrolimus cream $1 \%$ twice daily.

After sterilizing the lesion site, the patient was given local anesthesia for one hour using Xyla-P topical cream (Tehran Shimi, Tehran, Iran) under closed dressing, and subsequently, microneedling was performed. Then, the predetermined lesion site was prepared and draped.

Microneedling was conducted with Med Amiea revive (MT.DERM GmbH, Berlin, Germany). The needle penetration depth was initially set to $0.5 \mathrm{~mm}$ and then increased gradually until the lesion observed pinpoint bleeding. The microneedling procedure involves a combination of horizontal, vertical, and oblique device passes on the selected lesions, repeating approximately 3 to 4 times, till pinpoint bleeding has appeared (Figure 2). The pen was held vertically against the skin, and the bleeding spot was removed using sterile gauze impregnated with normal sterile saline. Since the microneedling was used for further penetration of topical cream, the treatment was performed just in the lesional area.

Microneedling is considered a noninvasive esthetic procedure with a low rate of associated side effects. But, skin irritation, mild erythema, swelling, dryness, and flaking of the skin could be reported [17]. Also, burning, stinging, itching, and swelling are considered as the most common side effects of pimecrolimus. So, it was instructed to the patients to report in case of any side effects [18].

2.3. Treatment Evaluation. The first step was to photograph patients using standardized global photography. All photos were taken with a digital camera (Canon IXUS 185 Digital Camera) in the same room and under the same lighting conditions regarding background, angle, and distance from the light source. Two independent dermatologists who were blinded to the study reviewed the baseline and posttreatment photos. The following qualitative responses to repigmentation were recorded: $(0 \%-25 \%)$ : mild improvement, $(26 \%-$ $50 \%)$ : moderate improvement, (51\%-75\%): good improvement, and (76\%-100\%): excellent improvement and follicular repigmentation [19].

Patients were evaluated using a patient satisfaction questionnaire, the dermatology quality of life index (DQLI) [8], and repigmentation responses to treatment as determined by two unbiased dermatologists blinded to the study [19].

2.4. Statistical Analysis. SPSS statistical software version 24 was used to analyze the data (IBM, Armonk, NY, USA). Mean and standard deviation were used to represent 


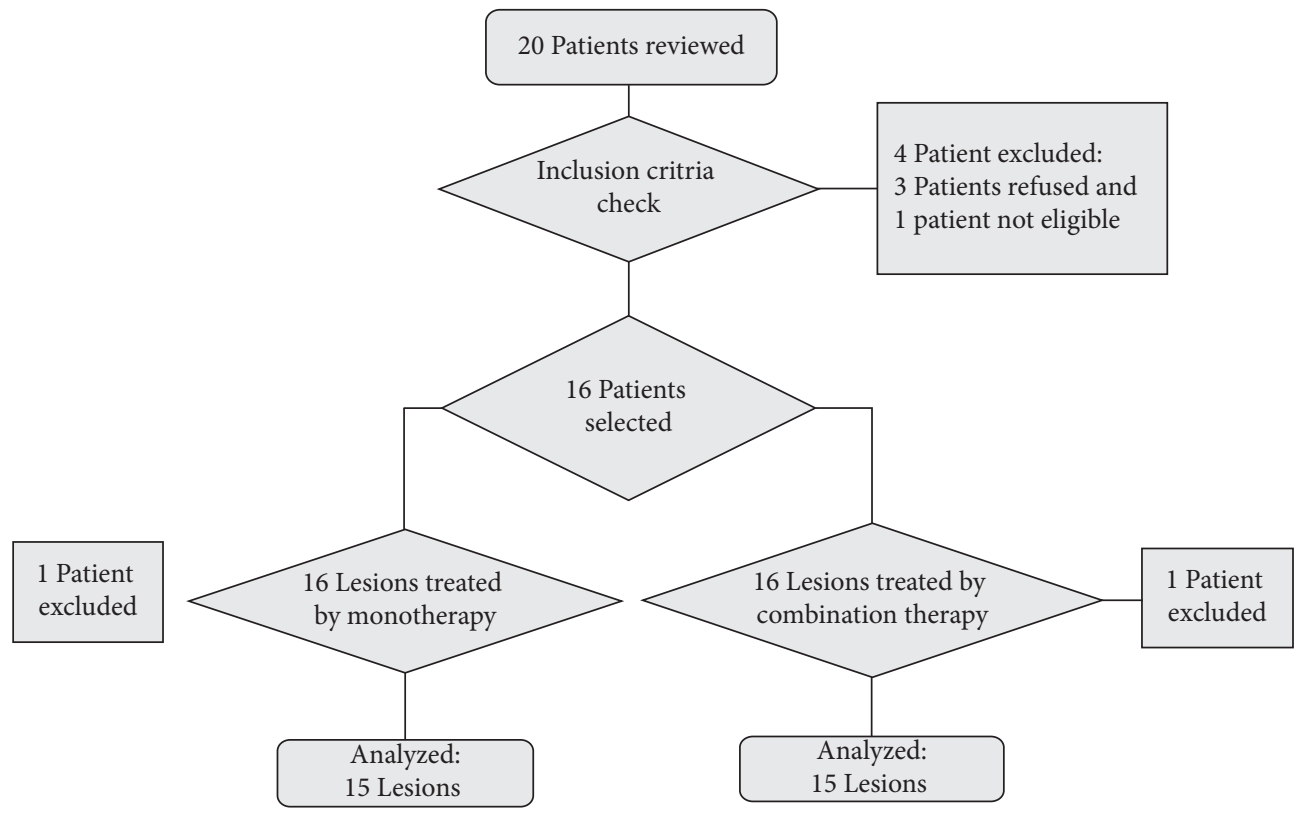

FIgUre 1: The flowchart of the study.

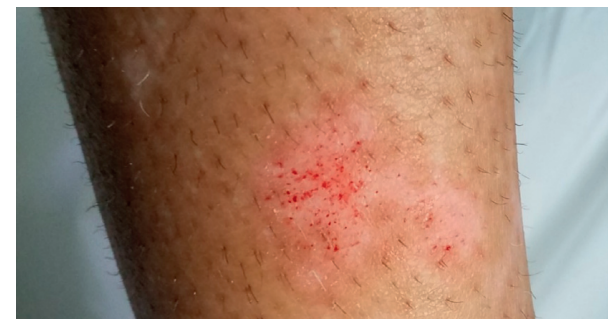

Figure 2: 20-year-old female patient's right leg: the appearance of pinpoint bleeding, the endpoint of the microneedling procedure.

quantitative data, while frequency or percentage represented qualitative data. The chi-square test was used to compare the data. $P$ values less than 0.05 were considered statistically significant in all analyses.

\section{Results}

Fifteen objects, eight (53.33\%) males and seven (46.66\%) females, were followed up in this intervention. Table 1 summarizes the patients' demographic characteristics.

Table 2 shows that no significant improvements were observed in the pimecrolimus method during the treatment or follow-up period (Figure 3), while in the combination therapy, the amount of repigmentation increased from the start to the end of the treatment and follow-up period. In combination therapy, we had poor recovery levels after two weeks of treatment and fair repigmentation reached to $40 \%$ after two months. Also, $33.3 \%$ of patients showed a good recovery status three months after treatment and excellent repigmentation appeared in $6.7 \%$ after 6 months' follow-up (Figure 4).

Friedman's test demonstrated that patients' recovery status improved significantly over time when microneedling and pimecrolimus were combined $(P<0.001)$. Fisher's exact
TABLE 1: Clinical data of studied cases.

\begin{tabular}{lccc}
\hline & & No. & $\%$ \\
\hline \multirow{2}{*}{ Sex } & Male & 8 & 53.33 \\
& Female & 7 & 46.66 \\
\hline \multirow{2}{*}{ Age (years) } & Mean \pm SD & $33.8 \pm 10.10$ & \\
\hline \multirow{2}{*}{ Family history } & Min-max & $18-56$ & \\
\hline \multirow{2}{*}{ Type of skin } & Negative & 12 & 80.00 \\
& Positive & 3 & 20.00 \\
\hline \multirow{2}{*}{ Previous history of treatment } & III & 10 & 66.66 \\
& IV & 5 & 33.33 \\
\hline
\end{tabular}

test revealed that the frequency distribution of patients' recovery levels was not significantly different between the two groups after one month of treatment $(P=0.999)$, but there was a noticeable difference at other points of time $(P<0.05)$.

As depicted in Table 3, no patient was satisfied with the pimecrolimus method from the start to the end of the study. In the combination method, there was no significant patients' satisfaction until 45 days after treatment. But, after 3 months' and 6 months' follow-up, $26.7 \%$ and $33.3 \%$ of respondents, respectively, reported a moderate and high level of satisfaction.

Friedman's test demonstrated that patient satisfaction with the combination of microneedling and pimecrolimus $(P<0.001)$ methods significantly increased over time. Fischer's exact test revealed a statistically significant difference in patient satisfaction at 45 days, two months, 75 days, three months, and six months following treatment in two groups.

Table 4 summarizes the numerical indices of patients' DLQI scores before treatment until the end of 6 months 
TABLE 2: Comparison between topical pimecrolimus therapy and combination therapy according to degree of repigmentation during the treatment and follow-up period.

\begin{tabular}{|c|c|c|c|c|c|c|}
\hline \multirow{2}{*}{$\begin{array}{l}\text { Treatment method } \\
\text { Repigmentation status }\end{array}$} & \multirow{2}{*}{$\begin{array}{l}\text { Pimecrolimus } \\
\text { Poor }\end{array}$} & \multicolumn{4}{|c|}{ Combination of microneedling and pimecrolimus } & \multirow{2}{*}{ Fischer's exact tes } \\
\hline & & Poor & Fair & Good & Excellent & \\
\hline After 2 weeks & $15(100 \%)$ & $15(100 \%)$ & $0(0 \%)$ & $0(0 \%)$ & $0(0 \%)$ & - \\
\hline After 1 month & $15(100 \%)$ & $14(93.3 \%)$ & $1(6.7 \%)$ & $0(0 \%)$ & $0(0 \%)$ & 0.999 \\
\hline After 45 days & $15(100 \%)$ & $11(73.3 \%)$ & $4(26.7 \%)$ & $0(0 \%)$ & $0(0 \%)$ & 0.027 \\
\hline After 2 months & $15(100 \%)$ & $9(60 \%)$ & $6(40 \%)$ & $0(0 \%)$ & $0(0 \%)$ & 0.01 \\
\hline After 75 days & $15(100 \%)$ & $6(40 \%)$ & $7(46.7 \%)$ & $2(13.3 \%)$ & $0(0 \%)$ & 0.001 \\
\hline After 3 months & $15(100 \%)$ & $6(40 \%)$ & $4(26.7 \%)$ & $5(33.3 \%)$ & $0(0 \%)$ & $<0.001$ \\
\hline After 6 months' follow-up & $15(100 \%)$ & $6(40 \%)$ & $4(26.7 \%)$ & $4(26.7 \%)$ & $1(6.7 \%)$ & $<0.001$ \\
\hline Friedman test & - & & & & & \\
\hline
\end{tabular}

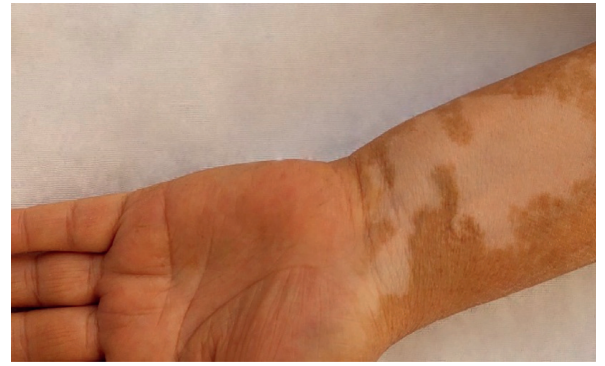

(a)

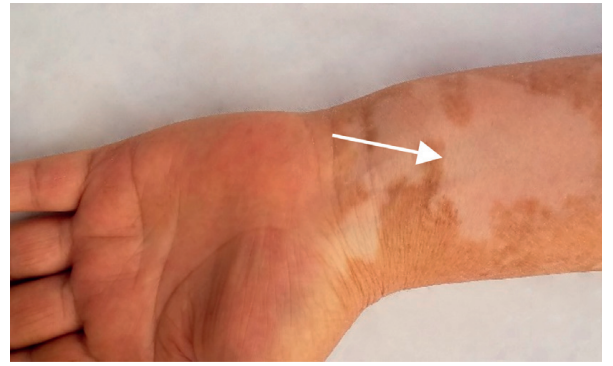

(b)

Figure 3: A 47-year-old female patient with refractory patches on the dorsal aspect of her left forearm: (a) before treatment; (b) six months' follow-up after treatment by topical pimecrolimus $1 \%$ on the pointed lesion. No changes were observed.

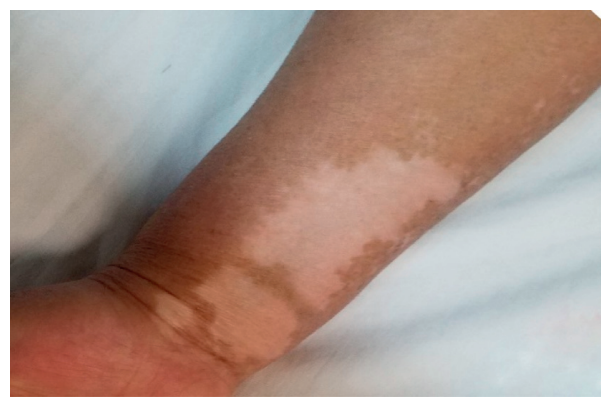

(a)

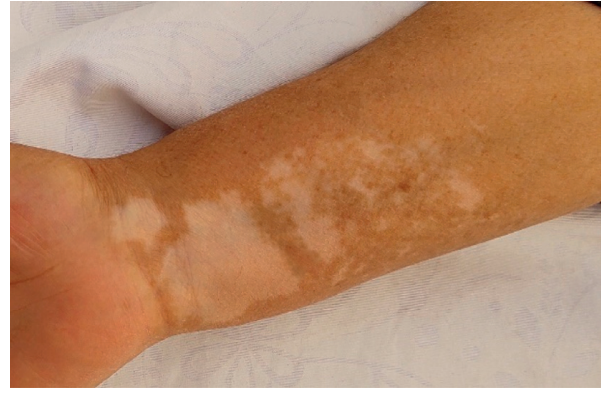

(c)

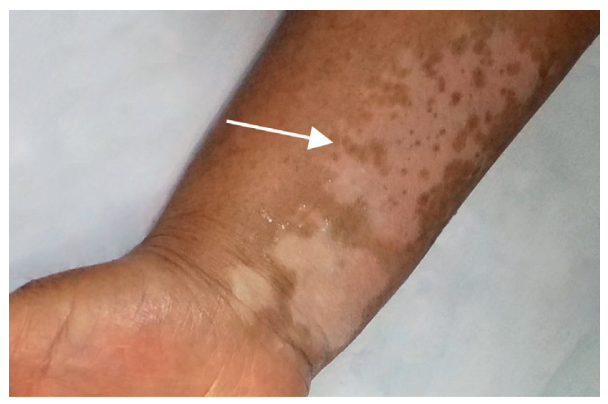

(b)

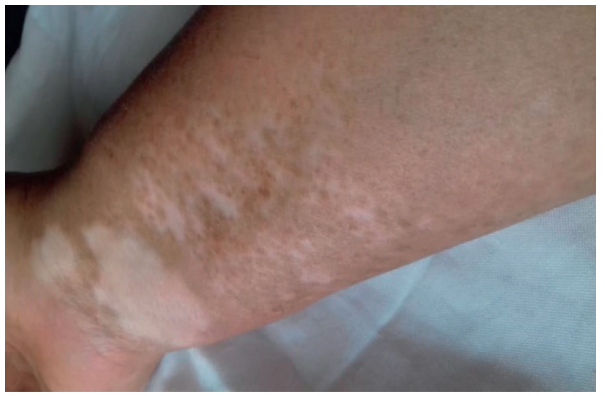

(d)

FiguRE 4: (a) A 47-year-old female patient with three refractory patches on the dorsal aspect of her right forearm, before treatment, (b) two months after treatment by microneedling plus topical pimecrolimus $1 \%$ on the pointed lesion (the largest lesion of the three), (c) three months after treatment with same procedure, and (d) at six months' follow-up after treatment.

of follow-up. The Kruskalovalis test indicated no significant difference in the DLQI scores of the two groups throughout the study $(P>0.05)$. Friedman's test revealed that when microneedling and pimecrolimus were used in combination, DLQI scores decreased significantly $(P=0.002)$.

There were no side effects reported in fourteen patients. Burning was observed in one patient following topical 
TABle 3: Percentage of patients' satisfaction in both groups during treatment and follow-up.

\begin{tabular}{|c|c|c|c|c|c|c|}
\hline \multirow{2}{*}{$\begin{array}{l}\text { Treatment method } \\
\text { Patients' satisfaction }\end{array}$} & \multirow{2}{*}{$\begin{array}{l}\text { Pimecrolimus } \\
\text { Ultralow }\end{array}$} & \multicolumn{4}{|c|}{ Combination of microneedling and pimecrolimus } & \multirow{2}{*}{ Fischer's exact test } \\
\hline & & Ultralow & Low & Moderate & High & \\
\hline After 2 weeks & $15(100 \%)$ & $14(93.3 \%)$ & $0(0 \%)$ & $1(6.7 \%)$ & $0(0 \%)$ & 0.999 \\
\hline After 1 month & $15(100 \%)$ & $13(86.6 \%)$ & $1(6.7 \%)$ & $1(6.7 \%)$ & $0(0 \%)$ & 0.762 \\
\hline After 45 days & $15(100 \%)$ & $10(6.66 \%)$ & $1(6.7 \%)$ & $4(26.7 \%)$ & $0(0 \%)$ & 0.035 \\
\hline After 2 months & $15(100 \%)$ & $9(60 \%)$ & $1(6.7 \%)$ & $5(33.3 \%)$ & $0(0 \%)$ & 0.019 \\
\hline After 75 days & $15(100 \%)$ & $6(40 \%)$ & $0(0 \%)$ & $7(46.6 \%)$ & $2(13.4 \%)$ & $<0.001$ \\
\hline After 3 months & $15(100 \%)$ & $6(40 \%)$ & $0(0 \%)$ & $4(26.7 \%)$ & $5(33.3 \%)$ & $<0.001$ \\
\hline After 6 months' follow-up & $15(100 \%)$ & $6(40 \%)$ & $0(0 \%)$ & $4(26.7 \%)$ & $5(33.3 \%)$ & $<0.001$ \\
\hline Friedman test & - & & & & & \\
\hline
\end{tabular}

TABLE 4: Comparison of numerical indices of patients' DLQI scores in monotherapy and combination therapy.

\begin{tabular}{|c|c|c|c|c|c|c|c|}
\hline \multirow{2}{*}{$\begin{array}{l}\text { Treatment method } \\
\text { DLQI }\end{array}$} & \multicolumn{3}{|c|}{ Pimecrolimus } & \multicolumn{3}{|c|}{$\begin{array}{l}\text { Combination of microneedling and } \\
\text { pimecrolimus }\end{array}$} & \multirow{2}{*}{ Kruskalovalis test } \\
\hline & $\begin{array}{c}\text { Mean standard } \\
\text { deviation }\end{array}$ & Median & $\begin{array}{c}\text { Interquartile } \\
\text { range }\end{array}$ & $\begin{array}{c}\text { Mean standard } \\
\text { deviation }\end{array}$ & Median & $\begin{array}{c}\text { Interquartile } \\
\text { range }\end{array}$ & \\
\hline Before treatment & $20.50(1.65)$ & 20.50 & $(22,19)$ & $20.73(1.83)$ & 21 & $(22,19)$ & 0.937 \\
\hline After 2 weeks & $20.64(1.59)$ & 21 & $(22,19)$ & $20.73(1.62)$ & 21 & $(22,19)$ & 0.953 \\
\hline After 1 month & $20.67(1.23)$ & 21 & $(22,20)$ & 20.33 (1.49) & 21 & $(22,19)$ & 0.812 \\
\hline After 45 days & $20.80(1.32)$ & 21 & $(21,20)$ & $20.20(2.04)$ & 20 & $(22,19)$ & 0.699 \\
\hline After 2 months & $20.67(1.49)$ & 21 & $(22,19)$ & $20.00(2.03)$ & 20 & $(21,18)$ & 0.492 \\
\hline After 75 days & $21.00(1.51)$ & 21 & $(22,20)$ & $19.07(2.57)$ & 19 & $(21,18)$ & 0.091 \\
\hline After 3 months & $20.80(1.47)$ & 20 & $(22,20)$ & $18.47(3.42)$ & 18 & $(22,15)$ & 0.132 \\
\hline $\begin{array}{l}\text { After } 6 \text { months' } \\
\text { follow-up }\end{array}$ & $20.80(1.74)$ & 21 & $(22,19)$ & $18.47(3.46)$ & 18 & $(22,15)$ & 0.149 \\
\hline Friedman test & & 0.943 & & & 0.002 & & \\
\hline
\end{tabular}

pimecrolimus, but the statistical analysis was not possible due to the complication's low frequency. No side effects were observed following the use of the microneedling device, and no infection, edema, or severe pain was reported.

\section{Discussion}

Vitiligo is a common, autoimmune disease that results in the destruction of the melanocytes and manifests as depigmented macules on various areas of the skin [20]. Despite the abundance of research and therapeutic modalities, there is still no cure for this condition [21, 22]. The patients' therapeutic approach should be personalized and tailored to their age, race, vitiligo subtype, affected skin area, and disease stability [23].

Topical calcineurin inhibitors (TCIs), such as tacrolimus and pimecrolimus, are widely used in vitiligo treatment [5]. On the other hand, the efficacy of microneedling for the treatment of vitiligo has been suggested in previous studies [24]. Microneedling is a minimally invasive procedure that has been used to treat a variety of dermatological conditions. Studies have shown that microneedling is a safe and effective treatment method for vitiligo, and its combination with other methods such as topical tacrolimus, topical calcipotriol, and 5-fluorouracil has demonstrated increased efficacy [24]. In the current study, we evaluated and compared the efficacy of topical pimecrolimus cream $1 \%$ alone with a combination of microneedling plus topical pimecrolimus $1 \%$ in the treatment of the patients with resistant vitiligo.
The efficacy of pimecrolimus alone cream for the treatment of the head and neck vitiligo has been shown by Boone et al. These researchers evaluated the efficacy and safety of pimecrolimus cream $1 \%$ in treating 26 patients with vitiliginous lesions in the head and neck and reported a median repigmentation rate of $72.9 \%$ after a six-month treatment period [25]. The present study demonstrated that combining microneedling with topical pimecrolimus effectively treats refractory stable vitiligo and improves patients' satisfaction. This higher response rate in the combination group is possibly due to the penetrance enhancer effect of microneedling for pimecrolimus as a synergetic effect or additive effect of the 2 treatment methods.

Dawid et al. reported pimecrolimus cream $1 \%$ ineffectiveness for treating body lesions of vitiligo. The authors reported that no improvement was observed in lesions after six months of treatment by topical pimecrolimus [26]. Consistent with this study, our findings showed none-efficacy of pimecrolimus cream alone for treatment of the limb lesions; however, the use of microneedling in addition to pimecrolimus provided a positive effect.

Mina et al. compared the efficacy of microneedling combined with 5-fluorouracil vs. microneedling combined with tacrolimus. The authors reported starting of the clinical repigmentation after three sessions of microneedling (i.e., within six weeks) [27]. In our study, as aforementioned, all patients in the pimecrolimus and pimecrolimus plus microneedling groups demonstrated a poor response within the first 45 days of the trial and it was after approximately 
two months that patients in the combination group showed clinical repigmentation.

A systematic review and meta-analysis showed that monotherapy with TCIs has favorable outcomes, with 55\% of patients achieving a mild response during a three-month treatment period. Additionally, at least $89.5 \%$ showed a mild response to the combination therapy of TCIs and phototherapy [28]. The results of our study also showed the superiority of the combination method over pimecrolimus monotherapy although instead of phototherapy, we used microneedling as the adjoint method.

In this study, the vitiligo lesions did not respond to topical pimecrolimus $1 \%$ monotherapy. This could be explained partly by the fact that these lesions were located on the extremities, which are more resistant to therapy than the rest of the body [29]. It should also be mentioned that we only selected lesions that were resistant to previous topical or systemic therapy.

Overall, our results showed the higher response rate of topical pimecrolimus plus microneedling combination versus pimecrolimus cream alone method as in terms of skin repigmentation. In addition, no significant side effect was observed in the patients of both groups that could be considered as a promising finding. To our best knowledge, this is the first study that evaluated a combination of microneedling plus pimecrolimus in the treatment of vitiligo. The present study demonstrated that combining microneedling with topical pimecrolimus effectively treats refractory stable vitiligo and improves patient satisfaction.

4.1. Study Limitation. Because of the COVID-19 pandemic, we had a small sample size.

\section{Conclusions}

Our study showed the efficacy and safety of microneedling plus pimecrolimus cream for the treatment of the resistant vitiligo lesions of the limbs. Further studies with a larger sample size are recommended to better evaluate the efficacy of this method.

\section{Data Availability}

The data used to support the findings of this study are included within the manuscript.

\section{Conflicts of Interest}

The authors declare no conflicts of interest regarding the publication of this paper.

\section{References}

[1] A. Asilian, S. Kazemipour, F. Mokhtari et al., "Effectiveness of dermabrasion plus 5-fluorouracil vs. suction blister in treating vitiligo: a comparative study," Dermatologic Therapy, vol. 34, no. 2, Article ID e14750, 2021.

[2] F. Iraji, E. Haftbaradaran, S. Davashi, Z. B. Azadeh, and B. J. Safoora, "Comparing the improvement of unstable vitiligo in patients treated by topical PUVA-therapy alone, topical
PUVA-therapy and oral vitamin D, and topical PUVA-therapy and oral vitamin D and vitamin B12," Journal f Isfahan Medical School (IUMS), vol. 34, no. 416, pp. 1699-1705, 2017.

[3] A. Alkhateeb, P. R. Fain, A. Thody, D. C. Bennett, and R. A. Spritz, "Epidemiology of vitiligo and associated autoimmune diseases in Caucasian probands and their families," Pigment Cell Research, vol. 16, no. 3, pp. 208-214, 2003.

[4] G. Iannella, A. Greco, D. Didona et al., "Vitiligo: pathogenesis, clinical variants and treatment approaches," Autoimmunity Reviews, vol. 15, no. 4, pp. 335-343, 2016.

[5] T. Passeron and J. P. Ortonne, "Vitiligo and other disorders of hypopigmentation," in Chapter No. 66 of Dermatology, Bolognia, Schaffer, and Cerroni, Eds., pp. 1087-1114, Elsevier, Amsterdam, Netherlands, 2018.

[6] F. Mohaghegh, A. Asilian, G. Faghihi, and N. Adibi, "A comparison between the efficacy of narrow band ultra violet $B$ phototherapy with and without needling of the lesion in the treatment of vitiligo," Journal of Research in Medical Sciences, vol. 17, no. Spec 1, pp. S131-S133, 2012.

[7] R. M. Halder and J. L. Chappell, "Vitiligo update," Seminars in Cutaneous Medicine and Surgery, vol. 28, no. 2, pp. 86-92, 2009.

[8] N. S. Sawant, N. A. Vanjari, and U. Khopkar, "Gender differences in depression, coping, stigma, and quality of life in patients of vitiligo," Dermatology Research and Practice, vol. 2019, Article ID 6879412, 10 pages, 2019.

[9] P. Redondo, A. Giménez de Azcarate, L. Marqués, M. GarcíaGuzman, E. Andreu, and F. Prósper, "Amniotic membrane as a scaffold for melanocyte transplantation in patients with stable vitiligo," Dermatology Research and Practice, vol. 2011, Article ID 532139, 6 pages, 2011.

[10] I. C. Poole, P. K. Das, R. M. J. G. J. Wijngaard, J. D. Bos, and W. Westerhof, "Review of the etiopathomechanism of vitiligo: a convergence theory," Experimental Dermatology, vol. 2, no. 4, pp. 145-153, 1993.

[11] F. Iraji, S. H. Banihashemi, G. Faghihi, Z Shahmoradi, $\mathrm{N}$ Tajmirriahi, and S. B Jazi, "A comparison of betamethasone valerate $0.1 \%$ cream twice daily plus oral simvastatin versus betamethasone valerate $0.1 \%$ cream alone in the treatment of vitiligo patients," Advanced Biomedical Research, vol. 6, p. 34, 2017.

[12] A. H. Siadat, N. Zeinali, F. Iraji et al., "Narrow-band ultraviolet $\mathrm{B}$ versus oral minocycline in treatment of unstable vitiligo: a prospective comparative trial," Dermatology Research and Practice, vol. 2014, pp. 1-4, 2014.

[13] S. Farajzadeh, I. Esfandiarpour, E. P. Khandani et al., "Efficacy of combination therapy of pimecrolimus $1 \%$ cream and mometasone cream with either agent alone in the treatment of childhood vitiligo," Journal of Pakistan Association of Dermatology, vol. 28, no. 4, pp. 507-513, 2019, http://jpad.com. pk/index.php/jpad/article/view/1315 p.

[14] D. J. Gawkrodger, A. D. Ormerod, L. Shaw et al., "Guideline for the diagnosis and management of vitiligo," British Journal of Dermatology, vol. 159, no. 5, pp. 1051-1076, 2008.

[15] Z. A. Ibrahim, G. F. Hassan, H. Y. Elgendy, and H. A. Alshenawy, "Evaluation of the efficacy of transdermal drug delivery of calcipotriol plus betamethasone versus tacrolimus in the treatment of vitiligo," Journal of Cosmetic Dermatology, vol. 18, no. 2, pp. 581-588, 2019, p.

[16] V. P. Zawar and G. M. Karad, "Needling in unresponsive stable vitiligo," Journal of the American Academy of Dermatology, vol. 75, no. 5, pp. e199-e200, 2016.

[17] T. S. Alster and P. M. Graham, "Microneedling: a review and practical guide," Dermatologic Surgery, vol. 44, no. 3, pp. 397-404, 2018, p. 
[18] M. Fox, Y. Helfrich, and S. Kang, "Other topical medications," Dermatology, pp. 2263-2277, Elsevier, Amsterdam, Netherlands, 2018.

[19] Z. Shahmoradi, F. Mokhtari, G. Faghihi, and N. Adibi, "Comparing the efficacy of topical clobetasol $0.05 \%$ plus 5 fluorouracil $5 \%$ cream vs. topical clobetasol $0.05 \%$ alone in the treatment of vitiligo," Journal of Research in Medical Sciences, vol. 17, pp. 7-12, 2012.

[20] A. Antonini, V. Leta, J. Teo, and K. R. Chaudhuri, "Outcome of Parkinson's disease patients affected by COVID-19," Movement Disorders, vol. 35, no. 6, pp. 905-908, 2020.

[21] F. Mokhtari, A. Bostakian, Z. Shahmoradi et al., "Potential emerging treatment in vitiligo using Er:YAG in combination with 5FU and clobetasol," Journal of Cosmetic Dermatology, vol. 17, no. 2, pp. 165-170, 2018.

[22] N. S. Patel, K. V. Paghdal, and G. F. Cohen, "Advanced treatment modalities for vitiligo," Dermatologic Surgery, vol. 38, no. 3, pp. 381-391, 2012.

[23] L. M. Felsten, A. Alikhan, and V. Petronic-Rosic, "Vitiligo: a comprehensive overview," Journal of the American Academy of Dermatology, vol. 65, no. 3, pp. 493-514, 2011, p.

[24] A. Salloum, N. Bazzi, D. Maalouf, and M. Habre, "Microneedling in vitiligo: a systematic review," Dermatologic Therapy, vol. 33, no. 6, p. 14297, 2020.

[25] B. Boone, K. Ongenae, N. Van Geel, S Vernijns, S De Keyser, and J. M Naeyaert, "Topical pimecrolimus in the treatment of vitiligo," European Journal of Dermatology, vol. 17, pp. 55-61, 2007.

[26] M. Dawid, M. Veensalu, M. Grassberger, and K Wolff, "Efficacy and safety of pimecrolimus cream $1 \%$ in adult patients with vitiligo: results of a randomized, double-blind, vehiclecontrolled study," Journal of the German Society of Dermatology, vol. 4, pp. 942-946, 2006.

[27] M. Mina, L. Elgarhy, H. Al-Saeid, and Z. Ibrahim, "Comparison between the efficacy of microneedling combined with 5-fluorouracil vs microneedling with tacrolimus in the treatment of vitiligo," Journal of Cosmetic Dermatology, vol. 17, no. 5, pp. 744-751, 2018.

[28] J. H. Lee, H. S. Kwon, H. M. Jung et al., "Treatment outcomes of topical calcineurin inhibitor therapy for patients with vitiligo," JAMA Dermatology, vol. 155, no. 8, pp. 929-938, 2019.

[29] V. Brazzelli, M. Antoninetti, S. Palazzini, T. Barbagallo, A. De Silvestri, and G. Borroni, "Critical evaluation of the variants influencing the clinical response of vitiligo: study of 60 cases treated with ultraviolet B narrow-band phototherapy," Journal of the European Academy of Dermatology and Venereology, vol. 21, no. 10, pp. 1369-1374, 2007. 